\title{
A STUDY ON QOS AWARE ROUTING IN WIRELESS MESH NETWORK
}

\author{
Arnab Kundu ${ }^{1}$, Suvajit Bhattacherjee ${ }^{2}$, Gairik Saha ${ }^{3}$ \\ ${ }^{1}$ Asst. Professor, Department of E.C.E., B.I.E.T., Suri, Dist.-Birbhum, PIN-731101, W.B., INDIA \\ ${ }^{2,3}$ Student, Department of E.C.E., B.I.E.T., Suri, Dist.-Birbhum, PIN-73101, W.B., INDIA \\ a.kunduwb@gmail.com, suvajit.ece.biet@gmail.com,gairik.biet@gmail.com
}

\begin{abstract}
Wireless Mesh Networks (WMN) mainly consists of mesh clients and mesh routers. It is mainly effective as an economically successful and a promising technology for providing wireless broadband services. Mesh routers basically form the backbone of the network $(W M N)$ and hence has the minimum mobility. They interconnect the mesh clients and the wireline backbone network. In order to approach the wireline level or same or near QoS, the Wireless Mesh Networks must be QoS aware. In this paper we have worked on the effect of variable transmission power to achieve the desired SNR and to maximize the number of active links at the same time for each links in the network and analyze different approaches to maintain the QoS routing of a wireless mesh network.
\end{abstract}

Index Terms:AODV; AQOR; DSDV; MANET; OPNET; QOS; WMN

\section{INTRODUCTION}

Wireless local area network is a process which provides Internet connectivity to mobile users, connection to the infrastructure. All the mobile users must reside within the basic service set (access point) with a radius up to 250 300meters. In relatively large areas we need to deploy a large number of access points (AP) to provide full coverage. In order to provide video-on-demand, online gaming and emergency communication services, end to end quality of services (QoS) must be up to that mark which is dependent on bandwidth. The QoS routing mechanism in a multi-hop wireless network faces some challenges due to the impact of interference. The available bandwidth in each an every node is dependent upon the interference occurred by its neighboring nodes within the interference range. Wireless mesh networks (WMNs) are easy to maintain, robust and provide reliable service coverage.

\section{WIRELESS MESH NETWORK}

WMNs consist of two types of nodes: mesh routers and mesh clients. Other than the routing capability for gateway/repeater functions as in a conventional wireless router, a wireless mesh router contains additional routing functions to support mesh networking. To further improve the flexibility of mesh networking, a mesh router is usually equipped with multiple wireless interfaces built on either the same or different wireless access technologies. Compared with a conventional wireless router, a wireless mesh router can achieve the same coverage with much lower transmission power through multihop communications.[1]. In this architecture, mesh clients represent other networks such as cellular, IEEE 802.11, IEEE 802.15 , and sensor networks. The gateways, mesh routers and the mesh clients form three-tier architecture [1], [2], [3].
$* * *$

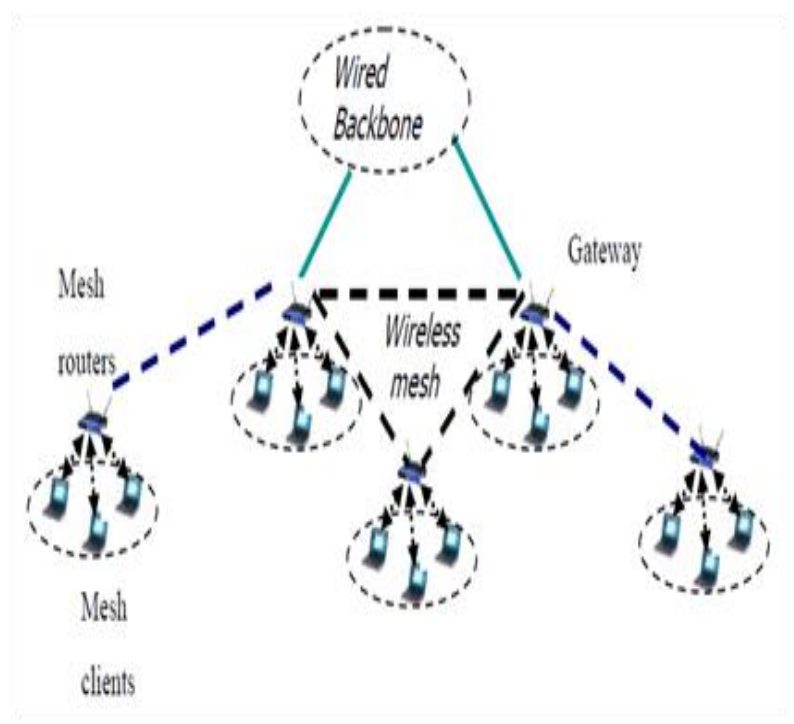

Fig1: Wireless Network Architecture

Unlike other distributed clustering schemes, ACE employs an emergent algorithm. Emergent algorithms much like artificial neural networks evolve to optimal solution through a mix of local optimization steps. The main idea of ACE is to allow a node to assess its potential as a $\mathrm{CH}$ before becoming one and stepping down if it is not the best $\mathrm{CH}$ at the moment. The algorithm works in iterations that do not have to be synchronized at the individual nodes. Spawning new clusters and migration of existing ones are the two functional components of ACE. A node spawns of new cluster when it decides to become a $\mathrm{CH}$. It broadcasts an invitation message to recruit its neighbors. Upon getting the invitation, a neighboring sensor joins the new cluster and becomes a follower of the new $\mathrm{CH}$. At any moment, a node can be a 
follower of more than one cluster. However, the node can be a loyal follower, i.e. a member, of only a single cluster.[3,5]

\section{APPLICATION}

WMNs offer a wide scope of applications including, but not limited to, providing broadband internet access, sharing information on goods and services, gaming, public safety, medical and emergency response, valuable asset security, neighborhood video surveillance, industrial monitoring. WMNs can also play an important role in disasters reporting and emergency networking.[4]On the small scale ([5], [6], [7], [8]), such as home or office, wireless mesh networking allows for connections to the internet as packet relays. Technologies such from anywhere, indoors or outdoors, without wires. Access points are placed in the transmission range of each other to act as IEEE $802.11 \mathrm{~b}$ or IEEE $802.11 \mathrm{a}$ is used to provide secure reliable, fast and wireless connectivity.

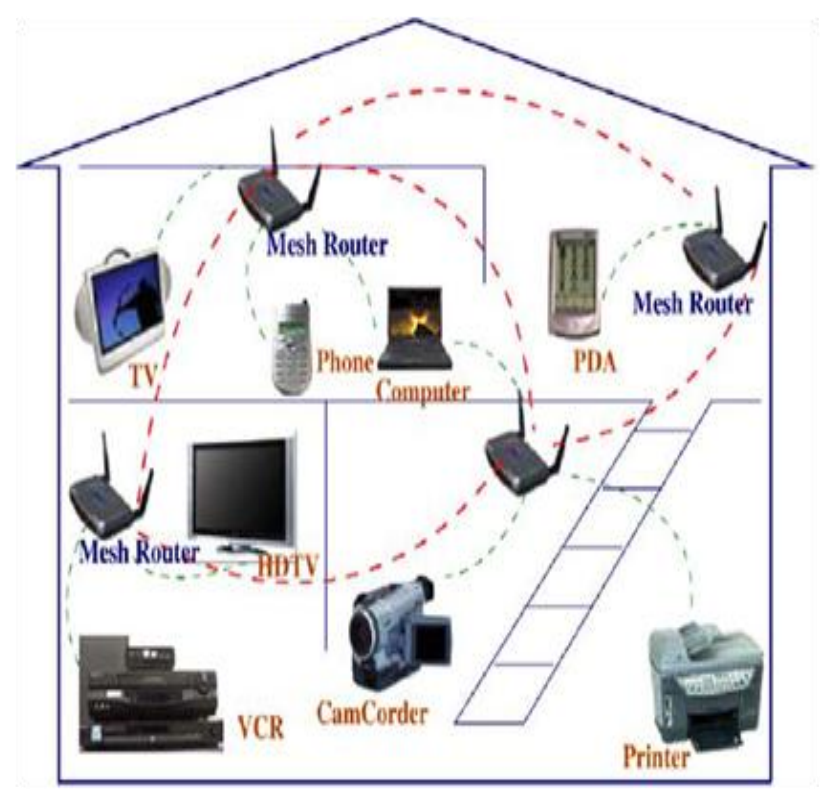

Computers with a wireless card can send and receive data to each other and to the Internet.These applications differ widely in their QoS requirements. One protocol designed to support one set of requirements may not be good for another set. Therefore designing protocols for a network should consider the different requirements, to provide the best performance for each set while achieving fair QoS among different sets.

\section{GENERAL ROUTING PROTOCOLS}

A route is scanned for QoS satisfaction in a procedure similar to route discovery in conventional routing protocols [4]. The route request packet is sent with QoS guarantee which is required on the route such as minimum required bandwidth. This packet compares its own measure packet with the metric fixed in its packet header. On occurrence of failure the packet is return to its source node. List of routes are for defining the route of the request packet. On failure the route repair procedure is also defined from the intermediate node where failure occurs.

\section{WIRELESS MESH ROUTING PROTOCOL (WMRP)}

WMR protocol has some salient features:

Topology discovery: Topology discovery procedure which maintains neighborhood information for each node by local information exchange. This procedure also provides each node the distance to the infrastructure, which facilitates the route discovery for external traffic.

Route discovery: Using the topology information obtained, route discovery algorithms can be introduced for both internal and external traffic.

Admission control with QoS constraints: For QoS guarantee required minimum bandwidth is to be provided and the maximum end-to-end delay. The method of measuring the required and available bandwidth as well as the end-to-end delay. The admission control module will use these measures to decide admission or rejection of a flow.

Bandwidth reservation: A temporary bandwidth reservation scheme which will help in the discovery and recovery of routes due to QoS violations.

Route recovery: Using the temporary bandwidth reservation scheme and neighborhood maintenance procedure we provide the route recovery procedure.

\section{QOS-AWARE ROUTING}

In modern communication systems providing QoS isa challenge to the service providers. Now a days multimedia service through the internet has flooded due to recent advances in communication technology. Service guarantee is specially required for video conferencing, distant learning and applications that required audio video transmission. This type of transmission is characterized by Variable Bit Rate (VBR). In this case allocation of bandwidth is a challenge, because if it is allocated according to the maximum rate resources are wasted and the other applications will be blocked. Several metrics are used to specify QoS requirements in networks such as minimum required throughput or capacity, maximum tolerable delay, maximum tolerable delay jitter and maximum tolerable packet loss ratio. An application may request guarantees on one or more metrics for its QoS. In the next sections, we present and discuss the different routing protocols developed to satisfy QoS requirements for traffic flows.[4]

\section{BACKGROUND AND LITERATURE SURVEY}

Routing and link scheduling are important components in satisfying the QoS requirements and utilizing the network bandwidth efficiently. They have long been addressed in wireless and wireline networks. However, few works have been done for WMNs. Power allocation has also been studied in wireless ad-hoc networks and sensor networks from the 
perspective of saving power. In the following, we review the literature of routing, link scheduling and power allocation to find the problems addressed and the impact of the proposed solutions in the WMNs. We mention here only the research done on WMNs for the routing and link scheduling. Since the power allocation is not addressed in WMNs we mention some work that was done in traditional ad-hoc networks.[4] As the above mentioned paper deals with frequency reuse, packet loss characteristics against traffic load, maximum throughput \& link scheduling, it is a superior approach for the better Qos routing in Wireless Mesh Network.

In 2005, Zhenget al. developed a graph-theoretic model to characterize the spectrum access problem and devised a set of heuristics to find high throughput and fair solutions.[9] Throughput is a parameter of Wireless Mesh Network. Since the previous paper deals with frequency reuse, packet loss characteristics against traffic load as well as maximum throughput, we better suggest the previous approach for the purpose of better QoS routing in WMN.

In 2003, Qi Xue and Aura Ganz proposed a novel ad hoc routing protocol, named Ad hoc QoS on-demand routing (AQOR).AQOR provides end-to-end QoS support regarding bandwidth and end-to-end delay. Qi and Aura has proposed a detail computation method that allows for measuring the endto-end delay in unsynchronized wireless environment. AQOR also provides QoS maintenance, destination initiated recovery process. [10] This paper introduced the use of AQOR protocol in MANETs for QoS maintenance purpose which is far reliable process as AQOR manages accurate admission control, resource reservation, estimates available bandwidth through efficient mechanism. It is also capable of handling end-to-end delay in unsynchronized Wireless Network.

In 2002, Qi Xue and Aura Ganz implemented wireless mesh routing protocol in Optimized Network (OPNET) Modeler. OPNET Modeler wireless suite is a network simulator which provides high fidelity modeling, simulation and analysis of a broad range of wireless communication. [11] The proposed network uses WMR which provides QoS support \& manages the infrastructure of wireless LANs as well as mobile Ad-hoc network using OPNET modeler.

In 2003, Elizabeth M. Belding-Royer Charles E. Perkins and Samir Das proposed Ad hoc On Demand Distance Vector(AODV) routing protocol, designed for mobile ad hoc networks which can deals with ten to thousands of mobile nodes. AODV is used for dynamic wireless networks i.e. nodes can enter and leave the network as per the requirement and to find the route to a particular destination node. The prime important point is that AODV never creates routing loops in its routing table. [12] As the distance vector routing follows some tables based on the distance vectors for any specific node in a network, it is capable to manage several nodes with a better QoS. But this proposed protocol only depends on routing vector table i.e. a single parameter of the network, the QoS of the following network may not be up to the mark.

In 1994, C.E. Perkins, P. Bhagwat examines the Destination Sequenced Distance Vector (DSDV) routing protocols for mobile ad hoc networks. The performance evaluation was done by using simulation tool NS2, NAM (Network Animator). Excel graph is used for preparing the graphs from the trace files. DSDV routing scheme for Ad hoc network is based on the BELLMAN-FORD algorithm. The routing loop problem has been solved by the application of this routing protocol. [13] The above discussed paper approaches the implementation of DSDV and it follows the Bellman-ford algorithm which contains a "negative cycle" and slower than dijkstra's algorithm. So this approach is not suitable for the network.

In 2004, Liliana CARRILlO, José L. MARZO, Pere VILÀ,César MANTILLAdescribed a hybrid routing scheme based on both an Ant Colony Optimization (ACO) and a Multi-Agent System (MAS) that 'pretends' to profit the advantages of both reactive and proactive algorithms.[14] This paper deals with ACO and MAS which is not the suitable option to solve the problem of QoS as this process does not follows any protocol or the parameters of the proposed network.

\section{CONCLUSIONS}

From the above mentioned survey, we have seen different approaches to maintain the QoS routing of a wireless mesh network. AQOR based QoS routing control was the basic method. After that AODV, DSDV routing protocol was proposed. Finally we can see the Ant Colony Optimization and Multi-agent System method for QoS aware routing protocol in a wireless mesh network. Here WMR protocol takes an important role to maintain the quality of services.

From the several approaches, I would like to prefer the method proposed by Tamer Abdelkader[4] which is based on three parameters of a network- Maximum throughput versus Minimum power, frequency reuse and packet Loss vs. traffic load. These methods are based on simulation procedure and the result from the simulation.

\section{ACKNOWLEDGEMENTS}

The authors would like to thank the authorities of Birbhum Institute of Engineering \& Technology for providing every kind of supports and encouragement during the working process 


\section{REFERENCES}

[1] I.F. Akyildiz, X.Wang, and W. Wang, “Wireless Mesh Networks: A Survey," Computer Networks Journal (Elsevier), March 2005.

[2] H. Jiang, W. Zhuang, X. Shen, A. Abdrabou, and P. Wang, "Differentiated services forwireless mesh backbone," IEEE Communication. Mag., vol. 44, no. 7, Jul. 2006, pp. 113-119. [3] H. Cheng, H. Jiang and W. Zhuang, "Distributed medium access control for wireless meshnetworks," Wireless Communications and Mobile Computing, vol. 6, no. 6, pp. 845-864,Sept. 2006.

[4] QoS Routing in Wireless Mesh Networks-By Tamer Abdelkader, A thesispresented to the University of Waterloo, Waterloo, Ontario, Canada, 2008

[5]“Mesh Dynamics website."http://www.meshdynamics.com [6]“BelairNetworkswebsite.” http://www.belairrnetworks.com [7]“'IntelWirelessMeshNetworkswebsite."http://www.intel.co $\mathrm{m} /$ update/contents/11032.h m

[8]“"NortelNetworkswebsite."http://www.nortelnetworks.com/ solutions/wrlsmesh/.

[9] H. Zheng and C. Peng, Collaboration and fairness in opportunisticspectrum access, Proceedings of IEEE ICC'2005, pp. 3132-3136.

[10]. Qi Xue and Aura Ganz. Ad hoc qos on-demandrouting (aqor) in mobile ad hoc networks. J. Parallel and Distributed Computing, pages 154-165, 2003.

[11]. Qi Xue and Aura Ganz. QoS routing for mesh-based wireless lans. In International Journal of Wirelss Information Networks, volume 9, July 2002.

[12] Elizabeth M. Belding-Royer Charles E. Perkins and Samir Das. Ad hoc on demand distance vector (aodv) routing. In IETF RFC 3561, 2003.

[13]. C.E. Perkins, P. Bhagwat, Highly dynamic destinationsequenced distance-vector routing (DSDV) for mobile computers, Computer Comm. Rev. 6 (1994) 234-244.

[14] MAntS-Hoc: A Multi-agent Ant-based System for Routing in Mobile Ad Hoc Networks Liliana CARRILLO, José L. MARZO , Pere VILÀ , César MANTILLA Institutd'InformàticaiAplicacions (IIiA), Universitat de Girona (UdG) Campus Montilivi, Av. LluísSantaló s/n, Edifici P-IV, 17071 Girona, Spain \{lilianac, marzo, perev, cmantill\}@eia.udg.es.

\section{BIOGRAPHIES}

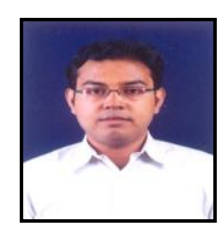

Arnab Kundu, born in India, obtained his M.Tech degree from JIS College of Engineering, Kalyani, West Bengal. Now he is Asst. Professor of E.C.E. Department in Birbhum Institute of Engineering \& Technology, P.O.:Suri, Dist.:Birbhum, PIN:731101, West Bengal, India. He is Life member of Indian Society for Technical Education, Indian Society of Remote Sensing, International Association of Engineers, The Institution of Electronics \& Telecommunication Engineers,
International Association of Engineers and Scientists (IAEST). E-Mail: a.kunduwb@gmail.com

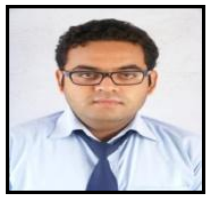

Suvajit Bhattacherjee, born in India. He is a final year UG student, Department of Electronics and Communication Engineering in Birbhum Institute of Engineering \& Technology, P.O.:Suri, Dist.:Birbhum, PIN:731101, West Bengal, India. He is a premium member of www.mywbut.com. Email: suvajit.ece.biet@gmail.com

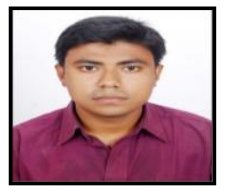

Gairik Saha, born in India. He is a final year UG student, Department of Electronics and Communication Engineering in Birbhum Institute of Engineering \& Technology, P.O.:Suri, Dist.:Birbhum, PIN:731101, West Bengal, India. $\mathrm{He}$ is a premium member of www.mywbut.com. Email: gairik.biet@gmail.com 